\title{
Scintillation array of the Experimental Complex NEVOD for EAS investigations
}

\author{
I.A. Shulzhenko ${ }^{1, a}$, M.B. Amelchakov ${ }^{1}$, N.S. Barbashina ${ }^{1}$, A.G. Bogdanov ${ }^{1}$, D.S. Brovtsev ${ }^{1}$, A. Chiavassa ${ }^{1,2,3}$, \\ N.E. Fomin ${ }^{1}$, D.M. Gromushkin ${ }^{1}$, N.N. Kamlev ${ }^{1}$, S.S. Khokhlov ${ }^{1}$, R.P. Kokoulin ${ }^{1}$, K.G. Kompaniets ${ }^{1}$, O.I. Likiy ${ }^{1}$, \\ V.V. Ovchinnikov ${ }^{1}$, A.A. Petrukhin ${ }^{1}$, O. Saavedra ${ }^{2}$, V.V. Shutenko ${ }^{1}$, and I.I. Yashin ${ }^{1}$ \\ 1 National Research Nuclear University MEPhI (Moscow Engineering Physics Institute), 115409 Moscow, Russia \\ 2 Dipartimento di Fisica dell' Università degli Studi di Torino, 10125 Torino, Italy \\ 3 Sezione di Torino dell' Istituto Nazionale di Fisica Nucleare - INFN, 10125 Torino, Italy
}

\begin{abstract}
In 2014, the creation of a new cluster type shower array NEVOD-EAS was started around the Cherenkov Water Detector NEVOD and coordinate detector DECOR. The shower array will allow to determine the size, axis position and arrival direction of EAS registered by these detectors. In 2015-2016, the NEVOD-EAS central part, including 4 clusters of scintillation detector stations located on an area of about $10^{4} \mathrm{~m}^{2}$, was deployed and started operation. This article presents the description of the NEVOD-EAS arrangement, detecting elements and data acquisition system, as well as the first results on EAS detection.
\end{abstract}

\section{Introduction}

For studying muon bundles in a wide range of zenith angles and energies with the complex of Cherenkov Water Detector (CWD) NEVOD [1,2] and coordinate detector DECOR [3], the method of local muon density spectra (LMDS) [4] has been developed. However, the estimations of primary particle energy using the LMDS technique has rather low accuracy $\left(\sigma\left(\lg E_{0}\right) \sim 0.4\right)$. It is due to the contribution of the extensive air showers (EAS) registered at different distances from the setup to the events with a fixed local muon density.

For an independent estimation of EAS parameters (arrival direction, size, axis position) in the energy range from $10^{15}$ to $10^{17} \mathrm{eV}$ using classical technique and, therefore, for calibration of the LMDS method and increasing its accuracy, a new cluster type shower array NEVOD-EAS is being constructed around the NEVOD-DECOR experimental complex.

\section{NEVOD-DECOR experimental complex}

The NEVOD-DECOR experimental complex (Figure 1) is a multi-purpose facility for effective registration of almost all components of cosmic rays at ground level. The setup is located in the National Research Nuclear University MEPhI (Moscow, Russia) in a special four-story building with a water reservoir (inner dimensions $9 \times 9 \times 26 \mathrm{~m}^{3}$ ) and is equipped with technical and technological systems necessary for the setup operation.

The basic installation of the complex is the CWD NEVOD. Its detecting system represents a spatial lattice of quasi-spherical modules (QSM). Each QSM includes six PMTs with flat photocathodes directed along the coordinate axes. The lattice of QSMs allows detection of Cherenkov radiation from all directions with practically equal efficiency and is formed by a set of vertical strings each containing 3 or 4 QSMs. The distances between the modules are $2.5 \mathrm{~m}$ along the detector, and $2.0 \mathrm{~m}$ across it and over the depth. Currently, the detecting system includes 91 QSMs (in total 546 PMTs). The signal readout is produced from the $12^{\text {th }}$ and $9^{\text {th }}$ dynodes that provides a wide dynamic range from 1 to $10^{5}$ photoelectrons and ensures operation in the calorimetric mode.

To calibrate the QSM photomultipliers during continuous experimental runs, the calibration telescope system (CTS) is used. This system contains 40 upper scintillation counters (on the water tank top) and 40 lower counters (on the tank bottom). Counter dimensions are $40 \times 20 \times$ $2 \mathrm{~cm}^{3}$. Vertical pairs of counters ensure calibration of PMTs of modules in four surrounding strings. Other combinations allow to obtain calibration curves for various distances from muon tracks.

The coordinate detector DECOR (see Fig. 1) is a system of plastic streamer tube chambers deployed around the CWD NEVOD. It includes eight vertically suspended eight-layer assemblies (supermodules) of such chambers with a total sensitive area of $70 \mathrm{~m}^{2}$. The chambers contain 16 tubes with $9 \times 9 \mathrm{~mm}^{2}$ inner cross section and $3.5 \mathrm{~m}$ length integrated in one PVC box. A special threecomponent gas mixture $\left(\mathrm{Ar}+\mathrm{CO}_{2}+\mathrm{n}\right.$-pentane $)$ provides operation of chambers in the limited streamer mode. Each layer of chambers is equipped with two-coordinate external strip readout system $(256 \mathrm{X}+256 \mathrm{Y}$ channels $)$ that allows to localize tracks with about $1 \mathrm{~cm}$ accuracy. The angular accuracy of track reconstruction is better than $0.7^{\circ}$ and $0.8^{\circ}$ for zenith and azimuth angles, respectively.

\footnotetext{
a e-mail: IAShulzhenko@mephi.ru
} 


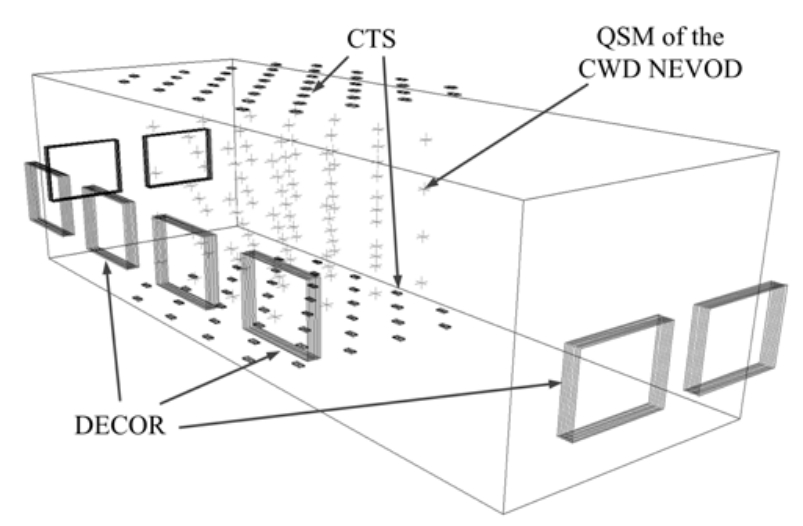

Figure 1. NEVOD-DECOR experimental complex.

\section{NEVOD-EAS shower array}

\subsection{Experimental arrangement}

In 2014, the creation of the NEVOD-EAS scintillation shower array was started. It is organized by the cluster principle and includes 12 independent clusters located around the experimental complex NEVOD-DECOR on the roofs of MEPhI laboratory buildings and on the ground. The layout of the NEVOD-EAS array is shown in Figure 2. Each cluster of the array consists of 16 scintillation counters of the EAS electron-photon component particles combined in 4 detector stations placed in the vertices of a rectangle with typical dimensions of about $15 \times 15 \mathrm{~m}^{2}$. The characteristic distance between the centres of the clusters is about $50 \mathrm{~m}$. The total area of the array is about $2 \times 10^{4} \mathrm{~m}^{2}$.

Since the clusters are located not in the same plane, but at different altitudes, new approaches to the EAS detection and organization of data acquisition system, as well as to experimental data analysis have been developed.

\subsection{Scintillation counters and detector stations}

Scintillation counters of the NEVOD-EAS array (see Fig. 2) were previously used in the KASCADE-Grande [5] (Germany) and EAS-Top [6] (Italy) experiments. A counter consists of plastic scintillator NE102A with the dimensions of $800 \times 800 \times 40 \mathrm{~mm}^{3}$ and photomultiplier Philips XP3462 which are installed inside the pyramidal housing. The distance between the PMT and scintillator is $30 \mathrm{~cm}$. The counter housing provides a possibility to install one additional PMT. The results of studies of the PMT and scintillator characteristics are presented in [7] and [8].

The detector station (DS) (see Fig. 2) of the array includes 4 scintillation counters. Three of the four DS counters are equipped with one ("standard") PMT for time and EAS particle density measurements. The standard PMT gain is adjusted for every counter in order to obtain the counter response to 1 particle (vertical equivalent muon, VEM) of about $13 \mathrm{pC}$. That provides a counter dynamic range of up to 100 particles. The fourth DS counter is equipped with two photomultipliers (a "standard" and an "additional" ones). The additional PMT ensures a wide linearity range at high particle densities $\left(\sim 10000\right.$ particles $\left./ \mathrm{m}^{2}\right)$ and has a gain 90 times lower than the standard one. The response of the additional PMT to 1 particle is about $0.15 \mathrm{pC}$. In the array, the DS counters are mounted inside special external housings. The total DS area is about $2.5 \mathrm{~m}^{2}$.

Detector stations also include a PMT power supply based on DC/DC-converter which transforms constant voltage $+12 \mathrm{~V}$ into high voltage in the range from -100 to $-2000 \mathrm{~V}$. For measuring ambient temperature and accounting for temperature effects on the scintillator light yield, the temperature sensor is installed inside the DS housing.

Analog signals from the DS counters are transferred to the cluster Local Post (LP) of primary data processing via coaxial cables.

\subsection{DAQ-system}

The data acquisition system of the NEVOD-EAS scintillation array is organized by a cluster principle and includes 2 main structural elements: the cluster and the Central DAQ Post (CP). The functional scheme of the NEVOD-EAS DAQ system is presented in Fig. 3.

\subsubsection{Cluster of the shower array}

Each cluster is an autonomous part of the setup which provides EAS registration, selection of events according to triggering conditions (registration thresholds, coincidence multiplicity and time gate) specified at the beginning of the experimental run, digitizing of amplitude information, timestamping of events and data transfer to the $\mathrm{CP}$ of the array. Basic elements of the NEVOD-EAS cluster are: the detecting elements (4 DS) and the Local Post of primary data processing.

Analog signals from the cluster detector stations (5 signals-4 from standard PMTs and 1 from additional one-per DS) are fed to the cluster LP. It includes: summator-multiplexor (SM), industrial I/O-module for the SM control and processing of temperature sensors' data, block of primary data processing (BPDP), some network equipment for communication with $\mathrm{CP}$ and several power supplies ensuring operation of all cluster elements. All LP electronics are enclosed inside the water-proof thermally stabilized box.

The BPDP digitizes DS signals, selects events according to various intra-cluster triggering conditions and transmits data to the Central DAQ Post. It includes two 2-channel boards of amplitude analysis (BAA) and a controller based on the FPGA Xilinx Spartan-6 which ensures all processor functions. Each BAA channel has 2 inputs: active (AI) and passive (PI). Signal digitizing is performed using a 12-bit fADC with a sampling frequency of $200 \mathrm{MHz}$. The registration threshold is set on the AI using 8-bit DAC. When the signal on the AI exceeds the registration threshold, the REQUEST signal is sent to the FPGA. If the number of REQUEST signals from BPDP measuring channels inside the specified time gate fulfils the specified multiplicity, FPGA sends a CONFIRMATION signal to the FIFO buffers of all channels. Then data are timestamped with an accuracy of $10 \mathrm{~ns}$, packed and sent to the CP.

Four-channel SM performs summation of analog signals from the standard PMTs. Each SM channel has 4 inputs and serves 1 DS. The summed signal from 4 standard PMTs of the DS is fed to the AI of the 


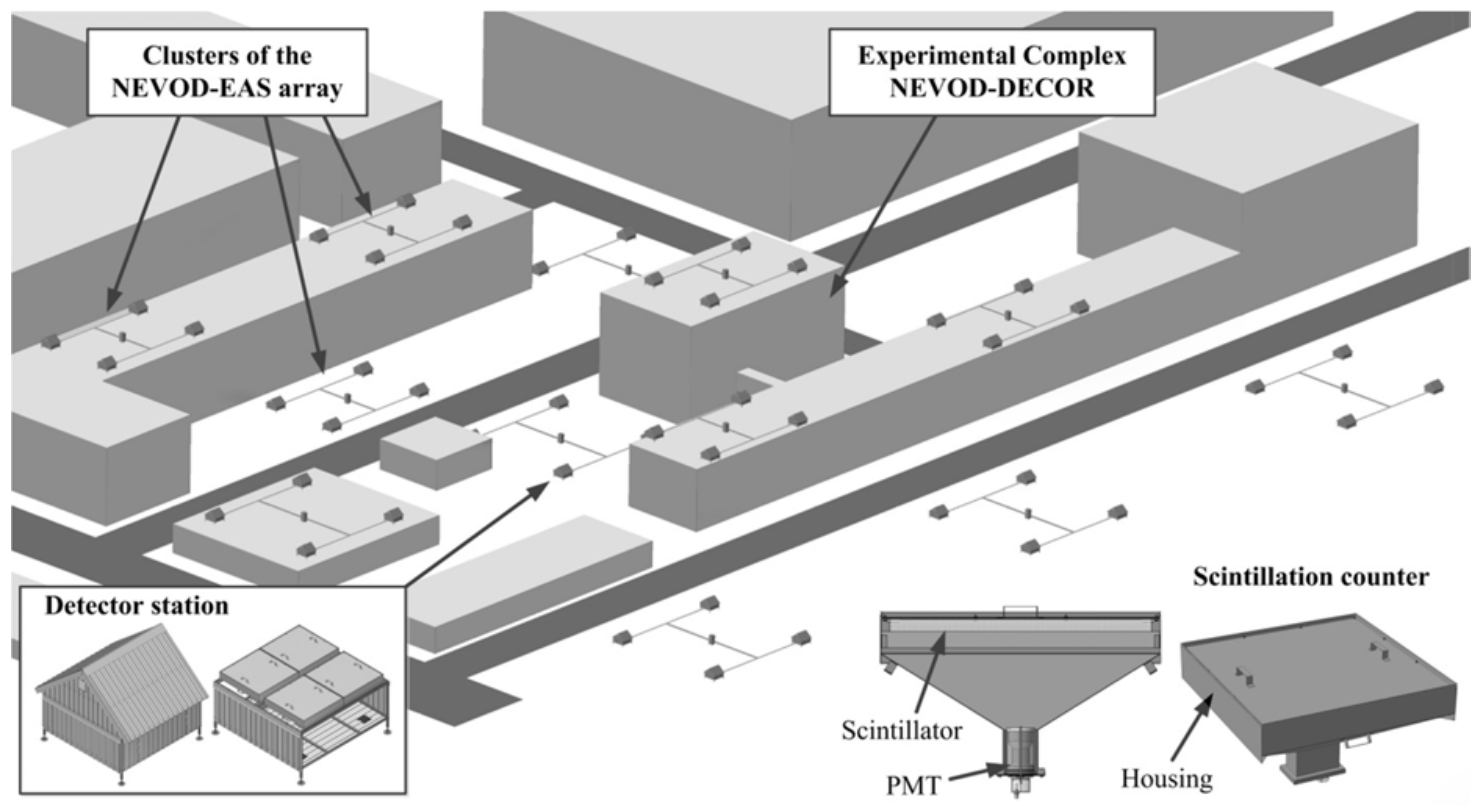

Figure 2. The layout of NEVOD-EAS shower array around the NEVOD-DECOR experimental complex. Left bottom corner: detector station housing and layout of counters inside the detector station. Right bottom corner: scheme of the counter.
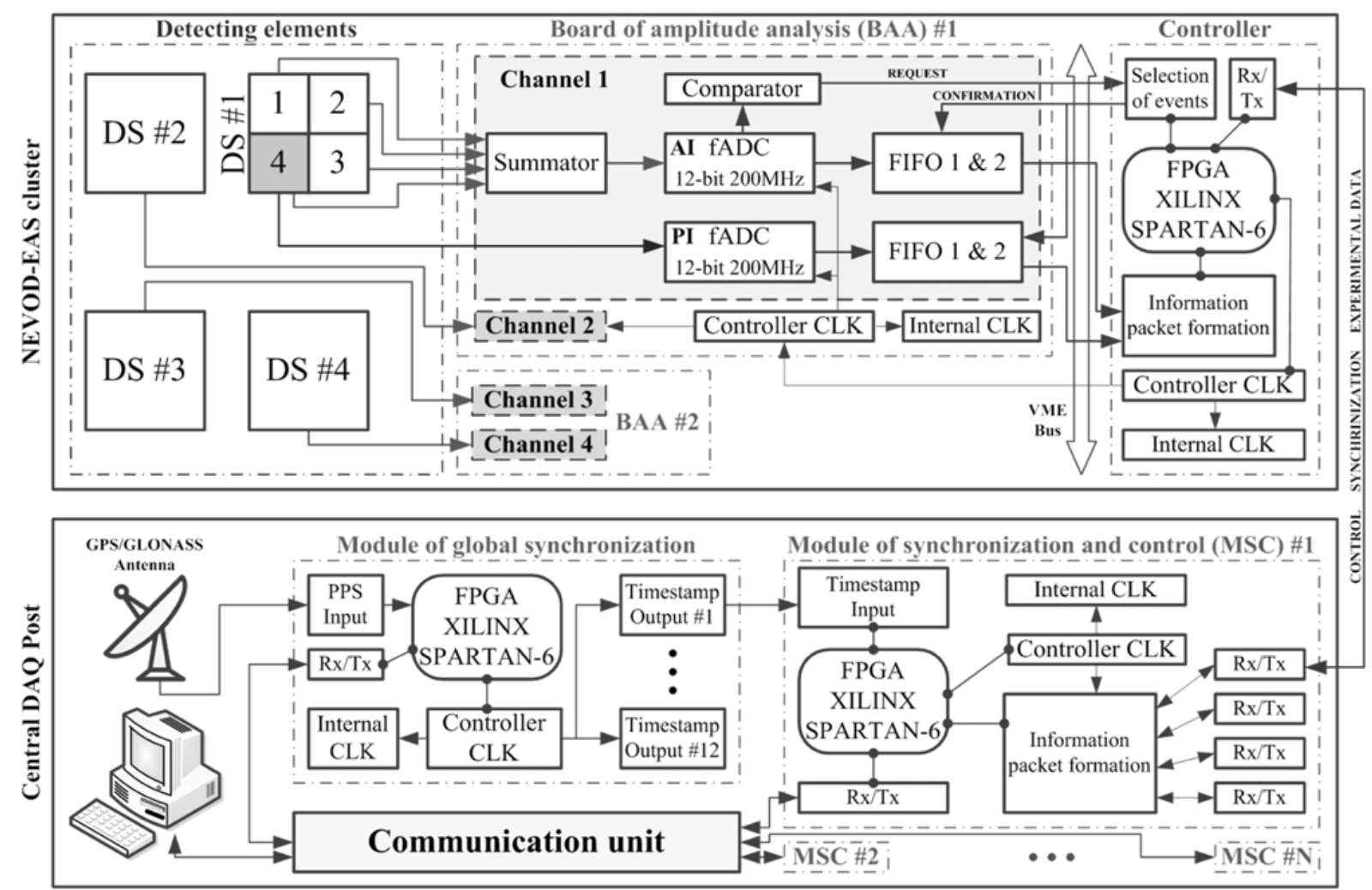

Figure 3. Functional scheme of the NEVOD-EAS shower array data acquisition system.

corresponding BAA channel. The additional PMT signal is fed to the corresponding PI of the BAA. The SM provides an opportunity to activate or deactivate defined inputs for measuring response spectra from the selected counter of the DS during the cluster operation monitoring. The SM is controlled using I/O-module which sets the mask of active SM inputs. This module has 8 analog inputs for the temperature sensors data.
All communications with the $\mathrm{CP}$ are performed using TCP/IP protocol via the fiber-optical line.

\subsubsection{Central $D A Q$ Post}

The Central DAQ Post ensures synchronization and control of all Local Posts of the NEVOD-EAS array, as well as the experimental and monitoring data reception 


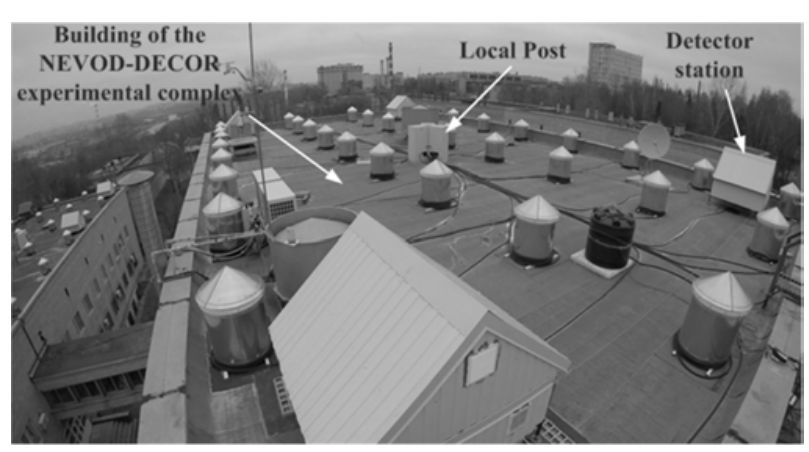

Figure 4. Photograph of the $3^{\text {rd }}$ cluster of the NEVOD-EAS array on the roof of the building of the NEVOD-DECOR experimental complex.

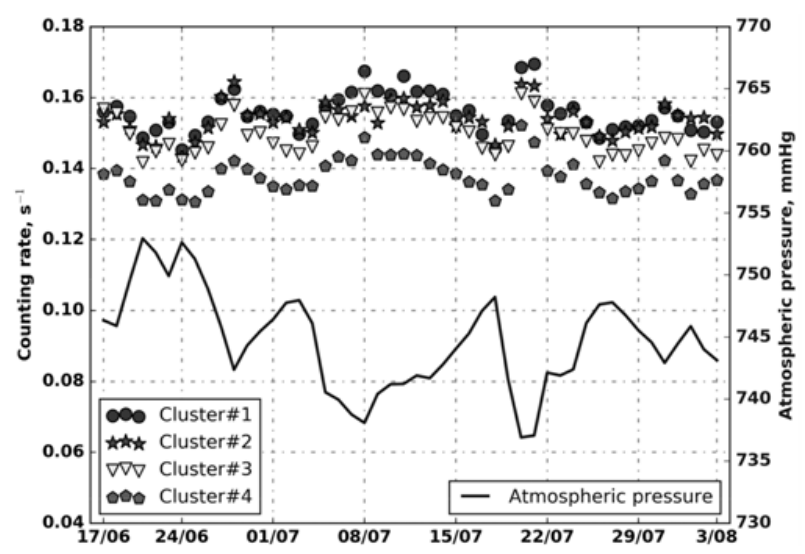

Figure 5. Counting rates of the NEVOD-EAS central part clusters and atmospheric pressure during the experimental run 17/06/16-03/08/16.

and storing. The CP (see Fig. 3) includes: personal computer (PC), GPS/GLONASS antenna, several modules of synchronization and control (MSC), module of global synchronization (MGS) and communication unit providing network connection between all $\mathrm{CP}$ elements.

Every MSC has 4 optical interfaces for the network connection to the BPDP of 4 NEVOD-EAS clusters and 1 Ethernet interface for the connection to central PC via the communication unit. At the beginning of an experimental run the specialized software on the central PC sends information packets to the MSC containing values of registration thresholds, coincidence multiplicities and time gates for 4 clusters. Then MSC broadcasts these options to certain clusters which configure their measuring channels and start registration. If one of the clusters registers an event, it sends information on the event time and contents of all ADC FIFO buffers to the MSC. The MSC, in its turn, adds information about the number of triggered clusters to the information packet and sends it to the central PC for processing and storing.

All electronics of the NEVOD-EAS array DAQ-system operate in the synchronous mode at the same clock frequency of $100 \mathrm{MHz}$. So, the synchronization accuracy of NEVOD-EAS clusters is $10 \mathrm{~ns}$.

Time synchronization of clusters is performed using the global positioning system (GPS/GLONASS) antenna and the MSC and MGS modules. The antenna which is directly connected to the MGS generates the PPS signal with a frequency of $1 \mathrm{~Hz}$. Upon receiving the PPS signal, the MGS send the synchronisation packet
Table 1. Dimensions and coordinates of the clusters of the NEVOD-EAS central part.

\begin{tabular}{|c|c|c|c|c|}
\hline $\begin{array}{c}\text { Cluster } \\
\text { No. }\end{array}$ & $\begin{array}{c}\text { Cluster } \\
\text { dimensions, } \mathrm{m}^{2}\end{array}$ & \multicolumn{3}{|c|}{$\begin{array}{c}\text { Coordinates of the } \\
\text { cluster centres, } \mathrm{m}\end{array}$} \\
\cline { 3 - 5 } & & $\mathrm{X}$ & $\mathrm{Y}$ & $\mathrm{Z}$ \\
\hline 1 & $13.3 \times 12.4$ & -28.4 & -7.8 & -7.0 \\
\hline 2 & $13.3 \times 12.4$ & -28.4 & 23.8 & -7.0 \\
\hline 3 & $25.1 \times 13.5$ & 0.0 & 0.0 & 0.0 \\
\hline 4 & $15.0 \times 15.0$ & 33.3 & 7.8 & -14.5 \\
\hline
\end{tabular}

containing the number of cycles of its internal clock to the special interfaces of the MSC modules. The MSC modules compare the obtained value to the number of cycles of their own internal clocks. Then MSC modules send the number of cycles of the MGS internal clock to the BPDP controllers of the clusters which also compare the obtained value to the number of cycles of their own and ADCs' internal clocks.

If some deviations are revealed during this comparison, all internal clocks of the NEVOD-EAS array DAQ-system are stopped. Upon the next PPS signal from the antenna all clocks are started again in the way described above.

The MSC also allows to measure time of the synchronization packet transmission from the CP to the LP of all clusters via optical cables. This value determines the time shift in the LP local time which had to be accounted for the correction of the cluster event time. All processor functions of the MSC and MGS modules are performed by the FPGA Xilinx Spartan-6.

\subsection{Central part of the NEVOD-EAS array}

In 2015-2016, the central part of the NEVOD-EAS scintillation array was created and started. The central part includes 4 clusters. Three clusters (1,2 and 3) are located on the roofs of MEPhI laboratory buildings and the $4^{\text {th }}$ one is on the ground surface. The total area of the NEVOD-EAS central part is about $10^{4} \mathrm{~m}^{2}$. The dimensions of clusters and their coordinates relative to the center of the roof of the NEVOD-DECOR building are presented in Table 1. The photograph of one of the NEVOD-EAS clusters is shown in Fig. 4.

The first continuous experimental run at the central part of the NEVOD-EAS array lasted 47 days (17/06/16$03 / 08 / 16$ ). The triggering condition for all clusters was a 4-fold coincidence of DS during the time gate with a duration of $120 \mathrm{~ns}$. Registration threshold for all detector stations was about $0.5-1.0$ particles (VEM). The counting rates of 4 central part clusters and the atmospheric pressure during this run are shown in Fig. 5. It is seen that the first 3 clusters have almost the same counting rates while the counting rate of the $4^{\text {th }}$ cluster is a little bit lower. This is due to its location close to one of the laboratory buildings which acts as a shield for EAS electron-photon component. The clear anti-correlation of the cluster counting rates and the atmospheric pressure can be observed. The estimated barometric coefficient is about $-0.8 \% / \mathrm{mmHg}$.

\subsection{EAS detection}

As the NEVOD-EAS array clusters are located not in the same plane, but at different altitudes, a special cluster approach to the reconstruction of registered extensive air 

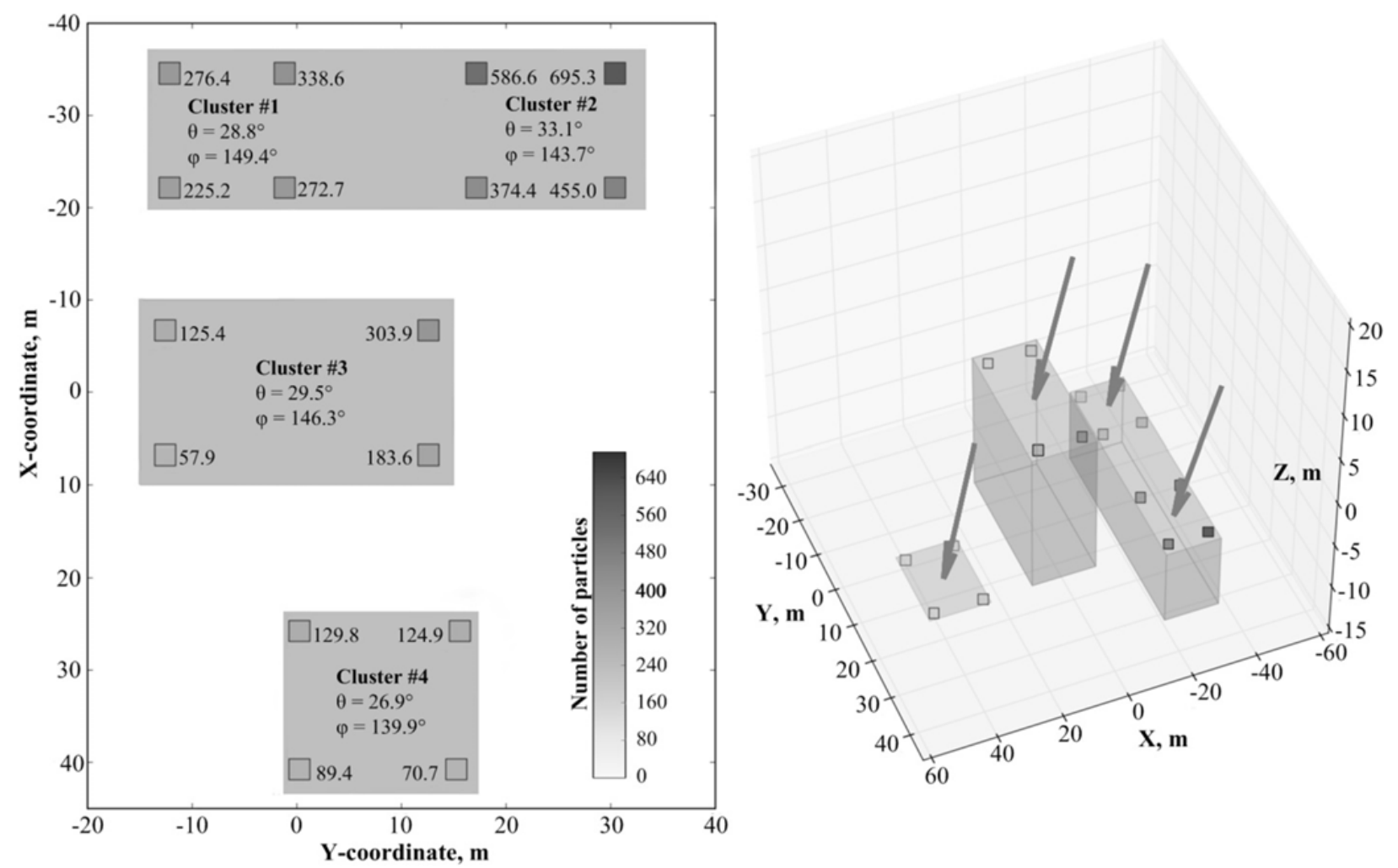

Figure 6. Reconstruction of the NEVOD-EAS array central part response to the extensive air shower.

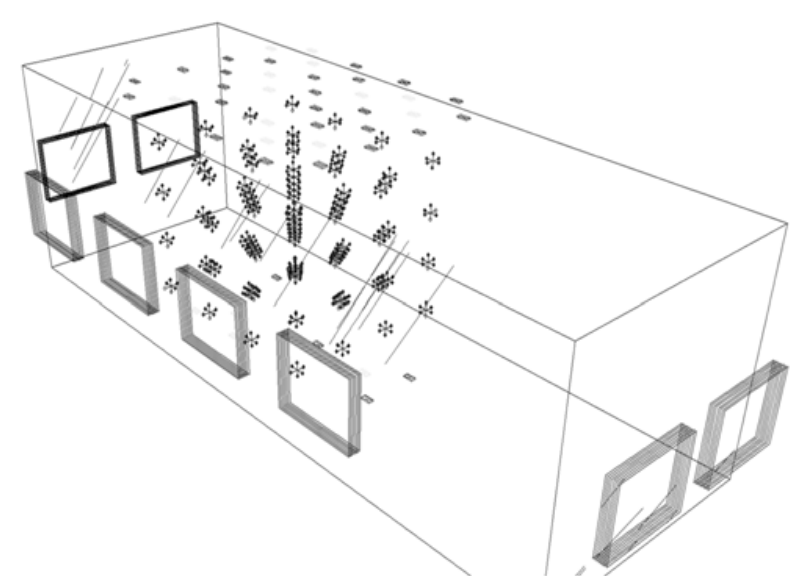

Figure 7. Reconstruction of the NEVOD-DECOR experimental complex response to the extensive air shower.

showers has been developed. In particular, the EAS arrival direction is determined in the following way. The events in which not less than 3 clusters have been triggered during the time interval of about $0.5 \mu$ s determined by the characteristic size of the setup $(\sim 100 \mathrm{~m})$ are selected. The intra-cluster triggering condition is a 4-fold coincidence of detector stations within a $120 \mathrm{~ns}$ time gate. For each cluster the arrival direction is calculated on the assumption of a flat EAS front. The resulting direction in the event is calculated by averaging directions reconstructed according to the data of triggered clusters.

The number of particles registered by each detector station is determined as the ratio between the DS response charge and its response charge to 1 particle (VEM, $\sim 13 \mathrm{pC}$ ). At high particle densities $\left(>100\right.$ particles $/ \mathrm{m}^{2}$ ) the data of the DS additional PMTs are taken into account.

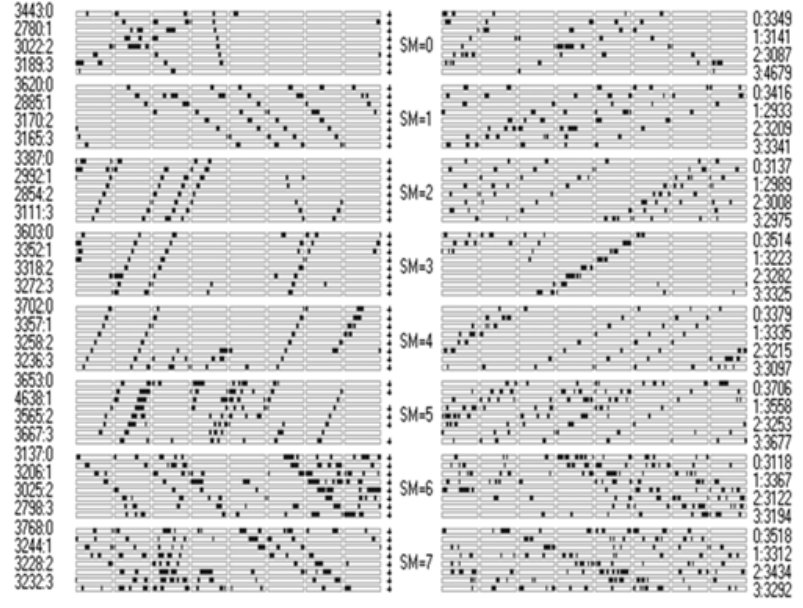

Figure 8. Response of the supermodules of the coordinate detector DECOR to the extensive air shower.

Figure 6 shows an example of the reconstruction of the NEVOD-EAS array central part response to the extensive air shower. Grey rectangles illustrate the cluster locations. Rectangles represent the detector stations. the number of particles registered by each DS is shown by the tone and printed near the rectangles. For all clusters the reconstructed azimuthal and zenith angles of the EAS arrival direction are indicated. The left part of Fig. 6 is the 3 -dimensional view of this event. The arrows correspond to the EAS front arrival direction reconstructed according to the data of each cluster. The highest particle density is observed in the DS \#1 of the $2^{\text {nd }}$ cluster. The particle density decreases in the direction from the $2^{\text {nd }}$ cluster to the $4^{\text {th }}$ one.

This extensive air shower has also been registered by the NEVOD-DECOR. Figure 7 shows geometric 
reconstruction of the experimental complex response. Crosses and circles represent triggered QSMs and PMTs of the CWD NEVOD, correspondingly. Rectangles are for the triggered counters of the CTS system. Straight lines show the tracks of muons reconstructed according to the data of the coordinate detector DECOR.

Figure 8 shows the response of the DECOR supermodules to this extensive air shower. Dark points represent triggered strips. In the left part of the figure are Y-strips (azimuth angle measurements) of supermodules. In the right part - X-strips (projected zenith angle measurements).

According to the data of the DECOR detector the azimuthal and zenith angles of the considered EAS arrival direction are $144.4^{\circ}$ and $29.5^{\circ}$, respectively. The results of the arrival direction reconstruction by the $3^{\text {rd }}$ cluster of the NEVOD-EAS array $\left(\varphi=146.3^{\circ}, \theta=29.5^{\circ}\right)$ are in a good agreement with these values.

\section{Conclusion}

In 2015-2016, the central part of the NEVOD-EAS cluster type shower array which includes 4 independent clusters (in total, 64 scintillation counters) located around the experimental complex of the CWD NEVOD and coordinate detector DECOR at the area of about $10^{4} \mathrm{~m}^{2}$ has been created and started.

The distributed structure of the NEVOD-EAS array DAQ-system allows to deploy registering elements of the setup even at different altitudes and provides implementation of a newly developed cluster approach to the reconstruction of EAS parameters. The first experimental run has proved the possibility of using of such a cluster approach.

This work was performed at the Unique Scientific Facility "Experimental complex NEVOD" with the support of the Ministry of Education and Science of the Russian Federation (grant RFMEFI59114X0002, MEPhI Academic Excellence Project 02.a03.21.0005 of 27.08.2013) and the Russian Foundation for Basic Research (grant 16-29-13028-ofi-m).

\section{References}

[1] V.M. Aynutdinov et al., Astrophysics and Space Science 258, 105 (1998)

[2] V.V. Kindin et al., Physics Procedia 74, 435 (2015)

[3] N.S. Barbashina et al., Instrum. Exp. Tech. 43, 743 (2000)

[4] R.P. Kokoulin et al., Nucl. Phys. B. (Proc. Suppl.) 196, 106 (2006)

[5] M. Aglietta et al., Nucl. Phys. B (Proc. Suppl.) 16, 493 (1990)

[6] A. Chiavassa et al., 28th ICRC, Tsukuba 2 (2003), p. 992

[7] I.A. Shulzhenko et al., Bull. Russ. Acad. Sci. Phys. 79, 389 (2015)

[8] I.I. Yashin et al., J. Phys.: Conf. Ser. 632, 012029 (2015) 